\title{
HOW FAST CAN THE CHORD LENGTH DISTRIBUTION DECAY?
}

\author{
YANN DEMICHEL,* Université Paris Ouest Nanterre La Défense \\ ANNE ESTRADE, ${ }^{* *}$ Université Paris Descartes \\ MARIE KRATZ,*** ESSEC Business School Paris \\ GENNADY SAMORODNITSKY, ${ }^{* * * *}$ Cornell University
}

\begin{abstract}
The modeling of random bi-phasic, or porous, media has been, and still is, under active investigation by mathematicians, physicists, and physicians. In this paper we consider a thresholded random process $X$ as a source of the two phases. The intervals when $X$ is in a given phase, named chords, are the subject of interest. We focus on the study of the tails of the chord length distribution functions. In the literature concerned with real data, different types of tail behavior have been reported, among them exponentiallike or power-like decay. We look for the link between the dependence structure of the underlying thresholded process $X$ and the rate of decay of the chord length distribution. When the process $X$ is a stationary Gaussian process, we relate the latter to the rate at which the covariance function of $X$ decays at large lags. We show that exponential, or nearly exponential, decay of the tail of the distribution of the chord lengths is very common, perhaps surprisingly so.
\end{abstract}

Keywords: Chord length; crossing; Gaussian field; bi-phasic medium; tail of distribution

2010 Mathematics Subject Classification: Primary 60K05

Secondary 60D05; 60G10; 60G55; 60G70

\section{Introduction}

Studying porous media, such as human bones, food, rocks, etc. leads naturally to $D$-dimensional Boolean models describing the presence or absence of material. Mathematically, a Boolean model is a function $f: \mathbb{R}^{D} \rightarrow\{0,1\}$, where the part of the space where the function $f$ takes value 0 represents the 'empty' part (lack of material, or 'pore'), while the part of the space where the function $f$ takes value 1 represents the 'full' part (presence of material, or 'matrix'). A Boolean model is often chosen to be stochastic, and a possible stochastic Boolean model is

\footnotetext{
Received 21 September 2009; revision received 27 September 2010.

* Postal address: MODAL'X, EA 3454, Université Paris Ouest Nanterre La Défense, 200 Avenue de la République, 92001 Nanterre Cedex, France. Email address: yann.demichel@u-paris10.fr

During the elaboration of this work, Yann Demichel was a member of MAP5, Université Paris Descartes.

** Postal address: MAP5, UMR CNRS 8145, Université Paris Descartes, 45 rue des Saints-Pères, 75270 Paris 06, France. Email address: anne.estrade@ parisdescartes.fr

*** Postal address: ESSEC Business School Paris, Avenue Bernard Hirsch BP 50105, 95021 Cergy Pontoise Cedex, France. Email address: kratz@essec.fr

Marie Kratz is also a member of MAP5, Université Paris Descartes.

**** Postal address: School of Operations Research and Information Engineering, Cornell University, Ithaca, NY 14853, USA. Email address: gennady@orie.cornell.edu
} 
obtained by thresholding a random field $\left(X_{t}\right)_{t \in \mathbb{R}^{D}}$ at a given level $\gamma$ :

$$
f(t)=\mathbf{1}_{(\gamma, \infty)}\left(X_{t}\right)= \begin{cases}1 & \text { if } X_{t}>\gamma \\ 0 & \text { otherwise }\end{cases}
$$

This procedure is very commonly considered by physicists (see [3], [13], and [15] for instance).

Here we adopt a one-dimensional point of view: we draw test lines through the random medium $\left(X(\boldsymbol{x}), \boldsymbol{x} \in \mathbb{R}^{3}\right)$, and, for any line $\Delta$, we identify $(X(\boldsymbol{x}), \boldsymbol{x} \in \Delta$ ) with a process $X=\left(X_{t}, t \in \mathbb{R}\right)$.

The successive intervals with $f(t)=0$ or $f(t)=1$ are called chords. The chords have been studied in the physics and mathematics literature; see, for instance, [16], [21, p. 82], [22, p. 44], and the references therein. In particular, the chord lengths have been investigated. In a previous paper [10] we analytically defined the chord length distribution functions. In this paper we focus on their rate of decay.

More precisely, let $X=\left(X_{t}\right)_{t \in \mathbb{R}}$ be a continuously differentiable, real strictly stationary process defined on some probability space $(\Omega, \mathcal{F}, \mathrm{P})$. If the process has a finite variance, we denote its covariance function by $\rho$. All processes considered in this paper have a finite variance and most of them will be Gaussian.

Assuming that the derivative of the process does not vanish on intervals of positive lengths, the chord lengths are well defined by

$$
L^{0}=\inf \left\{s>0: X_{s}=\gamma\right\}
$$

and

$$
L^{k+1}=\inf \left\{s>0: X_{s+L^{0}+\cdots+L^{k}}=\gamma\right\} \text { for } k \geq 0,
$$

where $\gamma$ is a given level (threshold).

Empirically, both exponential-like and power-like rates of decay of the distribution of the chord lengths have been observed on real data; see, for instance, Chapter 2 of [19]. Note that this refers to the rate of decay of the probability that a chord is very long. In this paper we investigate the effect of the memory in the thresholded process $X$ on the rate of decay of this probability. In the case of stationary Gaussian processes the memory is measured by the covariance function $\rho$. In Section 2 we investigate the tail of the distribution of the chord lengths statistically, on simulated data. We attempt to discriminate between light and heavy tails (of the chord lengths) both using the mean excess plot method as a graphical method and estimating the shape parameter of the associated generalized Pareto distribution. The numerical results obtained there motivate a probabilistic analysis developed in the next section. When dealing with stationary Gaussian processes having vanishing memory $(\rho \rightarrow 0$ at $\infty$ ), as is the case in the numerical examples, one of our main results (Theorem 3.1(ii)) shows that, for a thresholded Gaussian process with an exponentially fast decreasing covariance function, the tail of the distribution of the chord lengths decays exponentially fast as well. Perhaps, even more surprisingly, we prove that, for underlying Gaussian processes whose covariance function is only assumed to decay to 0 at any speed at all, the chord length distribution decays faster than any negative power function (Theorem 3.1(i)). This is also true for all $r$-mixing processes (Theorem 3.2). These theoretical results are proved in Section 3. In order to make them more intuitive, we first study the chord length distribution decay in the simple case whenever the thresholded process is $m$-dependent (Proposition 3.3). 


\section{Statistical analysis of simulated data}

In this section we aim to investigate statistically the behavior of the tail of the distribution of the chord lengths induced by thresholding a stationary Gaussian process. The purpose here is to both illustrate and motivate the bounds of the next section. To generate samples of chord lengths, we simulate the underlying Gaussian process. We consider the first two chord lengths, two different thresholds, and three different types of covariance function of the underlying process. (All MATLAB ${ }^{\circledR}$ codes, samples, and outputs are available on the webpage of Y. Demichel.)

\subsection{Simulation}

We start with outlying the simulation procedure. Given the covariance function $\rho$ of a zero-mean unit variance Gaussian process $X$, we simulate the process on a discrete subset $\left\{t_{1}, \ldots, t_{n}\right\}$ of a compact interval $I$. These discrete observations will be used to determine approximately the chords completed within the interval $I$. Note that we need to simulate a mean 0 and variance 1 Gaussian vector $\left(X\left(t_{1}\right), \ldots, X\left(t_{n}\right)\right)$ with a covariance matrix

$$
\boldsymbol{R}=\left(\rho\left(\left|t_{i}-t_{j}\right|\right)\right)_{1 \leq i, j \leq n} .
$$

As usual, the key is to obtain the square root of the covariance matrix. Since the Cholesky decomposition method is very expensive, we use the circulant embedding matrix method (see [7]). Recall that in this approach the covariance matrix $\boldsymbol{R}$ is embedded into a circulant matrix $\boldsymbol{C}$ whose eigenvectors are computed with a fast Fourier transform. The square root $\boldsymbol{R}^{1 / 2}$ is built from these eigenvectors. This method works if and only if the minimal eigenvalue $v^{-}$of $\boldsymbol{C}$ is positive and this property is difficult to ascertain a priori. In practice we choose an interval $I$ and a finite grid $\left\{t_{1}, \ldots, t_{n}\right\} \subset I$, and compute the matrix $C$ and the minimal eigenvalue $v^{-}$. These choices are crucial since a bad choice may yield an untractable circulant matrix $\boldsymbol{C}$. In order to have a good sample of chords, we have to consider both a large interval $I$ (to ensure that large chords are not missing) and a fine grid $\left\{t_{1}, \ldots, t_{n}\right\}$ (to ensure that small chords are not missing). In any case, we will miss all the chords that do not fall within the compact interval $I$.

Gaussian processes with various covariance functions have been simulated using this method and its extensions; see, for instance, [11], [17], and [18]. We have chosen three types of covariance function $\rho$ according to their speed of decay, to see if that would imply different types of chord length tail behavior. For further illustration, we consider the first two chord lengths. The different covariance functions $\rho$ and certain related properties are described in Table 1 . We have chosen the time interval $I=[0,3]$ and a grid of 6000 points. In each case the circulant embedding matrix method works since the minimum eigenvalue is positive, thus allowing us to obtain samples of the first two chord lengths $L^{0}$ and $L^{1}$ for a specified threshold.

TABLE 1: Covariance functions used for simulation. Here $\mathrm{P}_{11}(x)=1-\frac{22}{3} x^{2}+33 x^{4}-\frac{77}{2} x^{5}+\frac{33}{2} x^{7}-$ $\frac{11}{2} x^{9}+\frac{5}{6} x^{11}$

\begin{tabular}{clcc}
\hline Example & Speed of decay & Expression $\rho(x)$ & Eigenvalue $v^{-}$ \\
\hline 1 & Compact support & $\mathrm{P}_{11}(5 x) \mathbf{1}_{[0,1]}(5 x)$ & $6.8426 \times 10^{-10}$ \\
2 & Very fast & $\exp \left(-(5 x)^{2}\right)$ & $1.1827 \times 10^{-11}$ \\
3 & Polynomial & $\left(1+(6 x)^{2}\right)^{-4}$ & $2.7569 \times 10^{-11}$ \\
\hline
\end{tabular}




\subsection{Statistical analysis}

We perform statistical analysis on the samples of the first two chord lengths for each of the above three covariance functions. We use two different thresholds, $\gamma=0$ and $\gamma=1$. The approach we use is common in the extreme value theory (see, for instance, [9, p. 294]). First, we use a graphical method, the mean excess plot (MEP) to try to judge whether the chord lengths have light (i.e. exponentially fast decreasing) or heavy (i.e. hyperbolically fast decreasing) tails. Next, we fit a generalized Pareto distribution (GPD) to the upper part of the chord length distributions. The estimate from the considered sample parameters of the latter will then indicate either light or heavy tails of the chord length distributions.

Even though we presently apply these two methods to simulated data, the same approach could be used on real data as well.

2.2.1. A graphical method: the MEP. Recall that the excess cumulative distribution function (CDF) $F_{u}$ of a random variable $X$ over a threshold $u \in \mathbb{R}$ is defined in the peak-over-threshold (POT) approach as the CDF of $X-u$ conditioned on $X>u$, namely,

$$
F_{u}(x)=\mathrm{P}(X-u \leq x \mid X>u), \quad x \geq 0 .
$$

The corresponding mean excess function $e$ of $X$ is defined by

$$
e(u)=\mathrm{E}(X-u \mid X>u),
$$

whenever it exists.

The plot of the mean excess function $e$ is a useful graphical tool to help distinguish between heavy and light tails. For example, $e(u)=1 / \lambda$ for any $u$ if $X$ is exponentially distributed with parameter $\lambda$; heavy-tailed distribution functions have a mean excess function tending to $\infty$, typically along an asymptotically straight line; distribution functions with tails decaying faster than exponentially fast are characterized by a mean excess function tending to 0 .

In practice, we use the empirical MEP

$$
\left\{\left(X_{k, n}, e_{n}\left(X_{k, n}\right)\right): k \in\{1, \ldots, n-1\}\right\},
$$

where $X_{1, n} \leq \cdots \leq X_{n, n}$ are the order statistics of an $n$-sample $\left(X_{i}\right)_{1 \leq i \leq n}$, and $e_{n}(u)$ is the empirical mean excess function defined using the empirical cumulative distribution function by

$$
\frac{1}{N_{u}} \sum_{j \in I_{n}(u)}\left(X_{j}-u\right)
$$

with

$$
I_{n}(u)=\left\{j: 1 \leq j \leq n, X_{j}>u\right\} \quad \text { and } \quad N_{u}=\operatorname{card}\left(I_{n}(u)\right) .
$$

In the case of $u$ equal to one of the order statistics, this is equivalent to

$$
e_{n}\left(X_{k, n}\right)=\frac{1}{n-k} \sum_{j=k+1}^{n}\left(X_{j, n}-X_{k, n}\right) \text {. }
$$

2.2.2. Fitting a GPD to the excesses over a threshold. Pickands [14] proved that, for a sufficiently high threshold $u$, the excess $\mathrm{CDF} F_{u}$ of any random variable $X$ in a domain of attraction of an extreme value distribution can be well approximated by a GPD $G_{\xi, \sigma(u)}$, with a shape 
parameter $\xi$ and scale parameter $\sigma=\sigma(u)>0$ :

$$
G(y)=G_{\xi, \sigma(u)}(y)= \begin{cases}1-\left(1+\xi \frac{y}{\sigma(u)}\right)^{-1 / \xi} & \text { if } \xi \neq 0, \\ 1-\exp \left(-\frac{y}{\sigma(u)}\right) & \text { otherwise. }\end{cases}
$$

Here $y \geq 0$ if $\xi \geq 0$ and $0 \leq y \leq-\sigma(u) / \xi$ if $\xi<0$. Most of the 'textbook' random variables are in the domain of attraction of some extreme value distribution, and so the above approximation of the excess CDF is very general. The shape parameter $\xi>0$ arises when $X$ is heavy tailed, and $\xi \leq 0$ corresponds to light tails. Therefore, the parameters of the fitted GPD distribution provide information on the tails of $X$.

An important question is how to select an appropriate high threshold $u$; we choose it by plotting the empirical mean excess function and choosing $u$ in the range where the latter appears to be linear or stable.

The parameters of a GPD can be estimated via different methods. We will use the method of moments; see [12]. If the $\left(Y_{j}\right)_{1 \leq j \leq N_{u}}$ denote the excesses over a given threshold $u$ in a given sample, then the moments estimators of the parameters $\xi$ and $\sigma(u)$ of the approximating GPD are respectively given by

$$
\hat{\xi}=\frac{1}{2}\left(1-\frac{\bar{Y}^{2}}{S_{Y}^{2}}\right) \quad \text { and } \quad \hat{\sigma}=\widehat{\sigma(u)}=\bar{Y}\left(\frac{1}{2}+\frac{\bar{Y}^{2}}{S_{Y}^{2}}\right),
$$

where $\bar{Y}$ and $S_{Y}^{2}$ are the sample mean and variance of the excesses:

$$
\bar{Y}=\frac{1}{N_{u}} \sum_{i=1}^{N_{u}} Y_{i} \quad \text { and } \quad S_{Y}^{2}=\frac{1}{N_{u}-1} \sum_{i=1}^{N_{u}}\left(Y_{i}-\bar{Y}\right)^{2} .
$$

Provided that the shape parameter satisfies $\xi<\frac{1}{4}$, it can be shown by standard methods that the random vector $(\hat{\sigma}, \hat{\xi})$ is asymptotically normal with covariance matrix $\boldsymbol{A}$ satisfying, as the sample sizes increase,

$$
N_{u} \boldsymbol{A} \sim \Gamma=\frac{(1-\xi)^{2}}{(1-2 \xi)(1-3 \xi)(1-4 \xi)}\left(a_{i j}\right)_{1 \leq i, j \leq 2},
$$

where

$$
\begin{aligned}
& a_{11}=2 \sigma^{2}(u)\left(1-6 \xi+12 \xi^{2}\right), \\
& a_{22}=(1-2 \xi)^{2}\left(1-\xi+6 \xi^{2}\right),
\end{aligned}
$$

and

$$
a_{12}=a_{21}=\sigma(u)(1-2 \xi)\left(1-4 \xi-12 \xi^{2}\right),
$$

from which a confidence interval with asymptotic confidence level $\alpha$ can be deduced:

$$
\begin{aligned}
\left(\begin{array}{c}
\hat{\sigma} \\
\hat{\xi}
\end{array}\right)+\left(\frac{1}{N_{u}} \Gamma\right)^{1 / 2}\left(\begin{array}{l}
q((1-\alpha) / 2) \\
q((1-\alpha) / 2)
\end{array}\right) & \leq\left(\begin{array}{c}
\sigma(u) \\
\xi
\end{array}\right) \\
& \leq\left(\begin{array}{c}
\hat{\sigma} \\
\hat{\xi}
\end{array}\right)+\left(\frac{1}{N_{u}} \Gamma\right)^{1 / 2}\left(\begin{array}{c}
q((1+\alpha) / 2) \\
q((1+\alpha) / 2)
\end{array}\right) .
\end{aligned}
$$

Here $q(x)$ denotes the $x$ th quantile of the standard normal distribution. 


\subsection{Application to the chord lengths}

We generated samples of the first chord lengths $\left(L_{i}^{0}\right)_{1 \leq i \leq n}$ and the second chord lengths $\left(L_{i}^{1}\right)_{1 \leq i \leq n}$ of size $n=10000$ each.

In each case we started by plotting the empirical mean excess function, in order to judge whether it appears to increase linearly for large levels, or to decay to 0 . This was done for each of the three types of covariance and for the two chosen thresholds $\gamma=0$ and $\gamma=1$. Next, on each such MEP, we selected a level $u$ in the range where $e_{n}$ looks approximately linear or stable. It is well known that selecting a proper threshold $u$ is not an easy task as it implies a balance between bias and variance: too high a value of $u$ leads to too few exceedances and, consequently, high variance of the estimators, whereas too small a value of $u$ increases the bias of the estimators. The standard practice is to adopt as low a threshold as possible, subject to the limiting GPD model providing a reasonable approximation to the empirical tail. We assess this graphically as well.

Having selected a level $u$, we estimate the corresponding GPD parameters as in (2.1), with the associated asymptotic confidence intervals (CI), given in (2.2), at the confidence level 99\%.

The results obtained for the two first chord lengths, $L^{0}$ and $L^{1}$, are summarized in Table 2 for the threshold $\gamma=0$, and in Table 3 for the threshold $\gamma=1$. The corresponding empirical

TABLE 2: Statistical results for $L^{0}$ and $L^{1}$ with $\gamma=0$.

\begin{tabular}{cccrccccc}
\hline Example & $u$ & $\begin{array}{c}\alpha \text { such that } \\
u=q_{n}(\alpha)\end{array}$ & \multicolumn{1}{c}{$N_{u}$} & \multicolumn{1}{c}{$\hat{\xi}$} & $\hat{\sigma}$ & $\mathrm{CI}(\xi)$ & $\mathrm{CI}(\sigma)$ \\
\hline \multicolumn{7}{c}{ Chord length $L^{0}$} \\
\hline 1 & 0.41 & $90 \%$ & 910 & -0.0955 & 0.1816 & $(-0.2531,0.0621)$ & $(0.1047,0.2585)$ \\
2 & 0.90 & $86 \%$ & 1374 & -0.1530 & 0.4575 & $(-0.3301,0.0241)$ & $(0.3169,0.5981)$ \\
3 & 0.49 & $91 \%$ & 881 & -0.1058 & 0.2286 & $(-0.2767,0.0651)$ & $(0.1343,0.3229)$ \\
\hline \multicolumn{8}{c}{ Chord length $L^{1}$} \\
\hline 1 & 0.35 & $83 \%$ & 1696 & -0.0005 & 0.1721 & $(-0.0953,0.0843)$ & $(0.1302,0.2140)$ \\
3 & 1.27 & $92.5 \%$ & 746 & -0.1911 & 0.4207 & $(-0.4526,0.0704)$ & $(0.2238,0.6176)$ \\
3 & 0.46 & $86 \%$ & 1380 & -0.0063 & 0.2056 & $(-0.1088,0.0962)$ & $(0.1530,0.2582)$ \\
\hline
\end{tabular}

TABLE 3: Statistical results for $L^{0}$ and $L^{1}$ with $\gamma=1$.

\begin{tabular}{|c|c|c|c|c|c|c|c|}
\hline Example & $u$ & $\begin{array}{l}\alpha \text { such that } \\
u=q_{n}(\alpha)\end{array}$ & $N_{u}$ & $\hat{\xi}$ & $\hat{\sigma}$ & $\mathrm{CI}(\xi)$ & $\mathrm{CI}(\sigma)$ \\
\hline \multicolumn{8}{|c|}{ Chord length $L^{0}$} \\
\hline 1 & 0.85 & $83 \%$ & 1700 & -0.1252 & 0.5437 & $(-0.2784,0.0280)$ & $(0.4099,0.6775)$ \\
\hline 2 & 1.95 & $90 \%$ & 1030 & -0.5614 & 0.5503 & $(-1.1079,-0.0149)$ & $(0.1420,0.9586)$ \\
\hline 3 & 1.22 & $88.25 \%$ & 1175 & -0.2812 & 0.6561 & $(-0.5701,0.0077)$ & $(0.3898,0.9224)$ \\
\hline \multicolumn{8}{|c|}{ Chord length $L^{1}$} \\
\hline 1 & 0.41 & $90.5 \%$ & 934 & -0.0576 & 0.5032 & $(-0.2263,0.1111)$ & $(0.3614,0.6450)$ \\
\hline 2 & 0.98 & $91.3 \%$ & 866 & -0.4243 & 0.9689 & $(-0.9412,0.0926)$ & $(0.4357,1.5421)$ \\
\hline 3 & 0.52 & $92.1 \%$ & 793 & -0.1025 & 0.6391 & $(-0.3210,0.1160)$ & $(0.4305,0.8477)$ \\
\hline
\end{tabular}



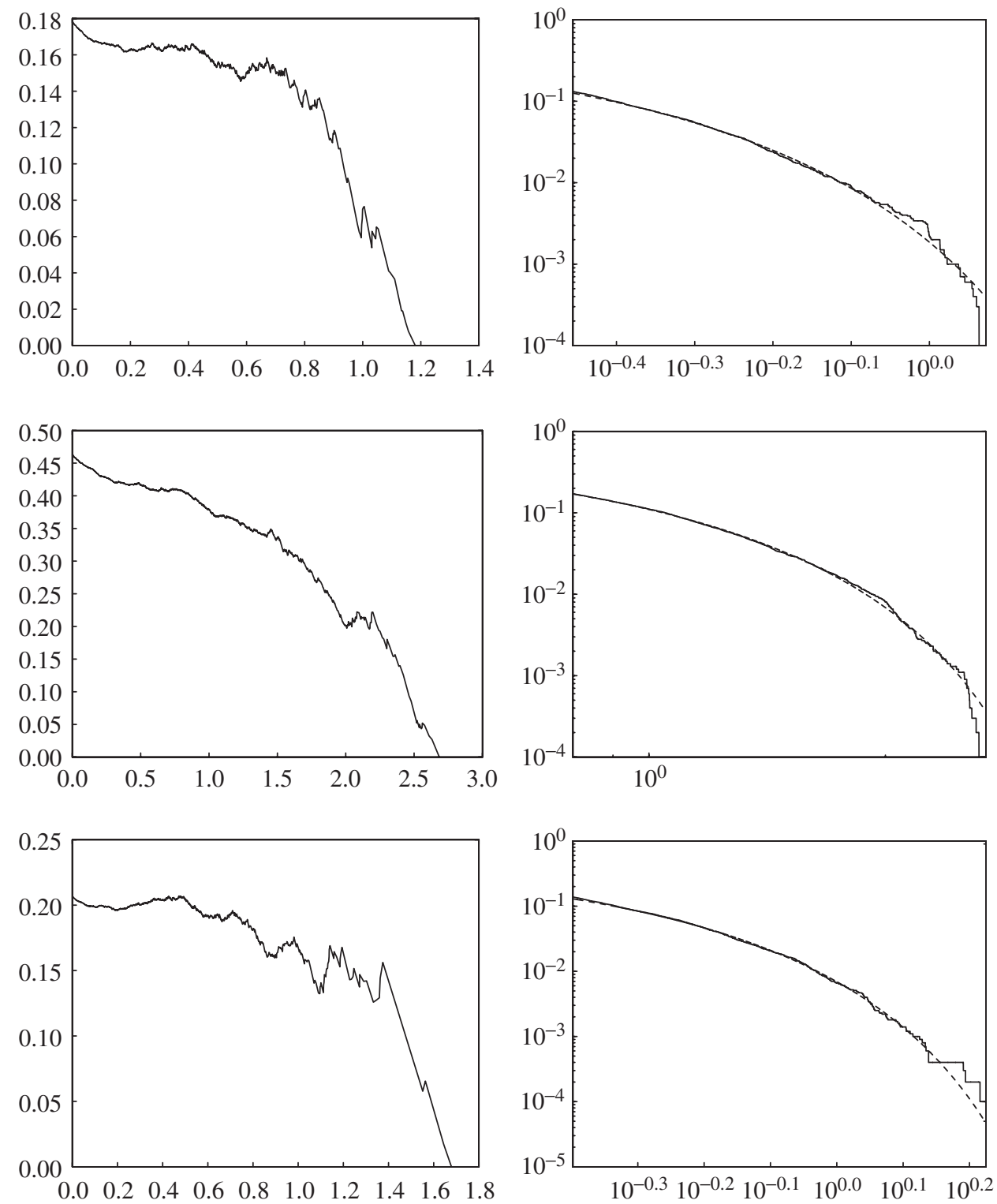

Figure 1: Length of the chord $L^{0}$ at threshold $\gamma=0$ for examples 1, 2, and 3 (in order from top to bottom) of the covariance function $\rho$. Left: the empirical mean excess plots. Right: log-log plots of the tails of the sample (solid lines) and approximating GPD (dashed lines) distribution functions. The parameters are given in the top half of Table 2.

MEPs, and the comparison of the tail of the empirical CDG and the tail of the approximating GPD appear in Figures 1-4. The empirical quantile function of order $\alpha$ is denoted by $q_{n}(\alpha)$.

It is obvious that the empirical mean excess functions (the left-hand plots of Figures 1-4) appear to be decaying to 0 , which is consistent with light tails of the chord length distributions. Furthermore, the estimated values of the shape parameter $\xi$ of the approximating GDP are all 

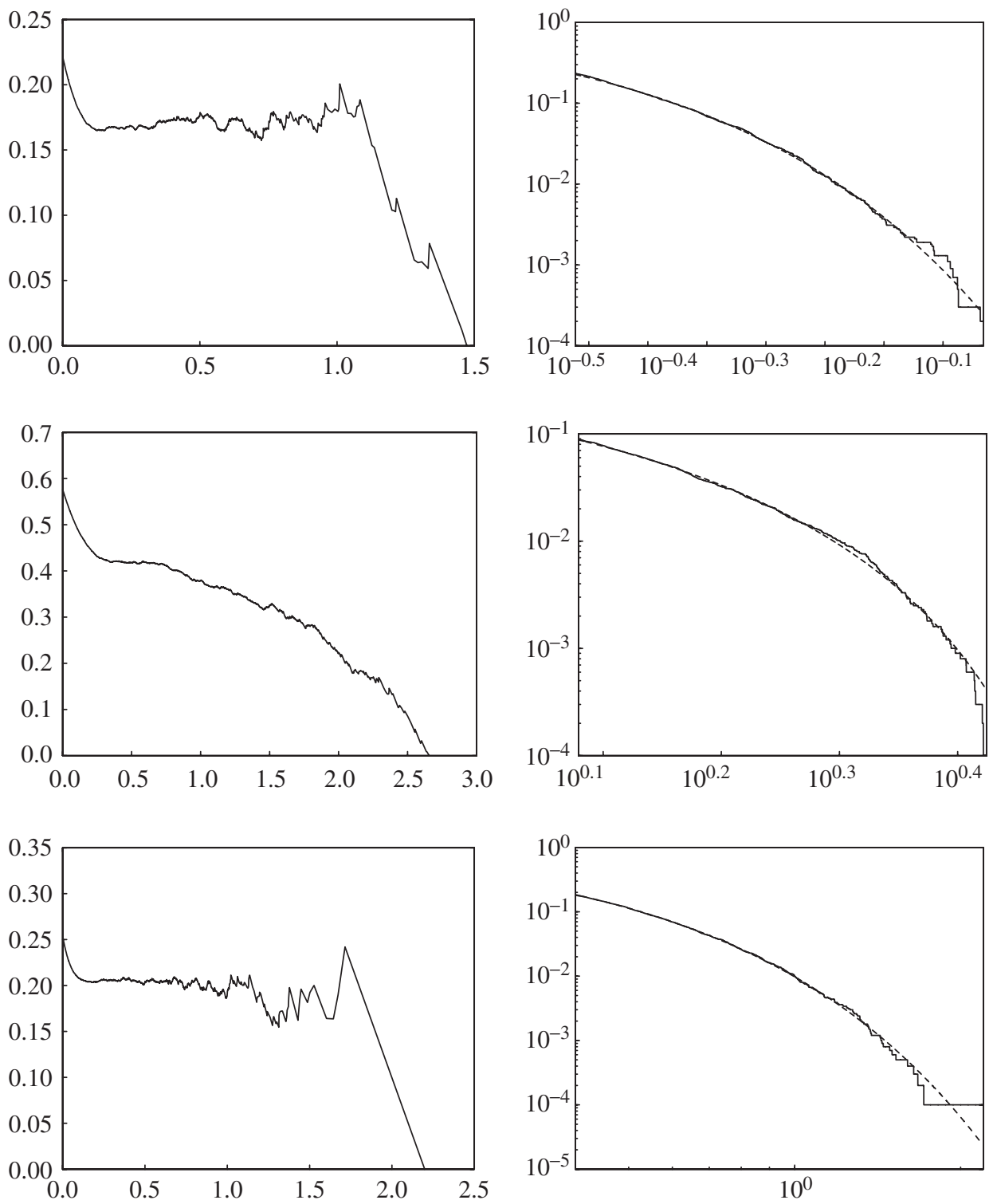

Figure 2: Length of the chord $L^{1}$ at threshold $\gamma=0$ for examples 1, 2, and 3 (in order from top to bottom) of the covariance function $\rho$. Left: the empirical mean excess plots. Right: log-log plots of the tails of the sample (solid lines) and approximating GPD (dashed lines) distribution functions. The parameters are given in the bottom half of Table 2.

negative. Even though most of the $99 \%$ confidence intervals for $\xi$ contain the origin, it is clear that the estimated shape parameter points towards light tails of the chord length distributions as well. The CDF of the sample and its approximating GPD are given in the right-hand plots of Figures 1-4. The nonlinear behavior of the log-log plots rules out the hypothesis of a power-law decay. The results do not seem to differ significantly for the two chord lengths, for the two 

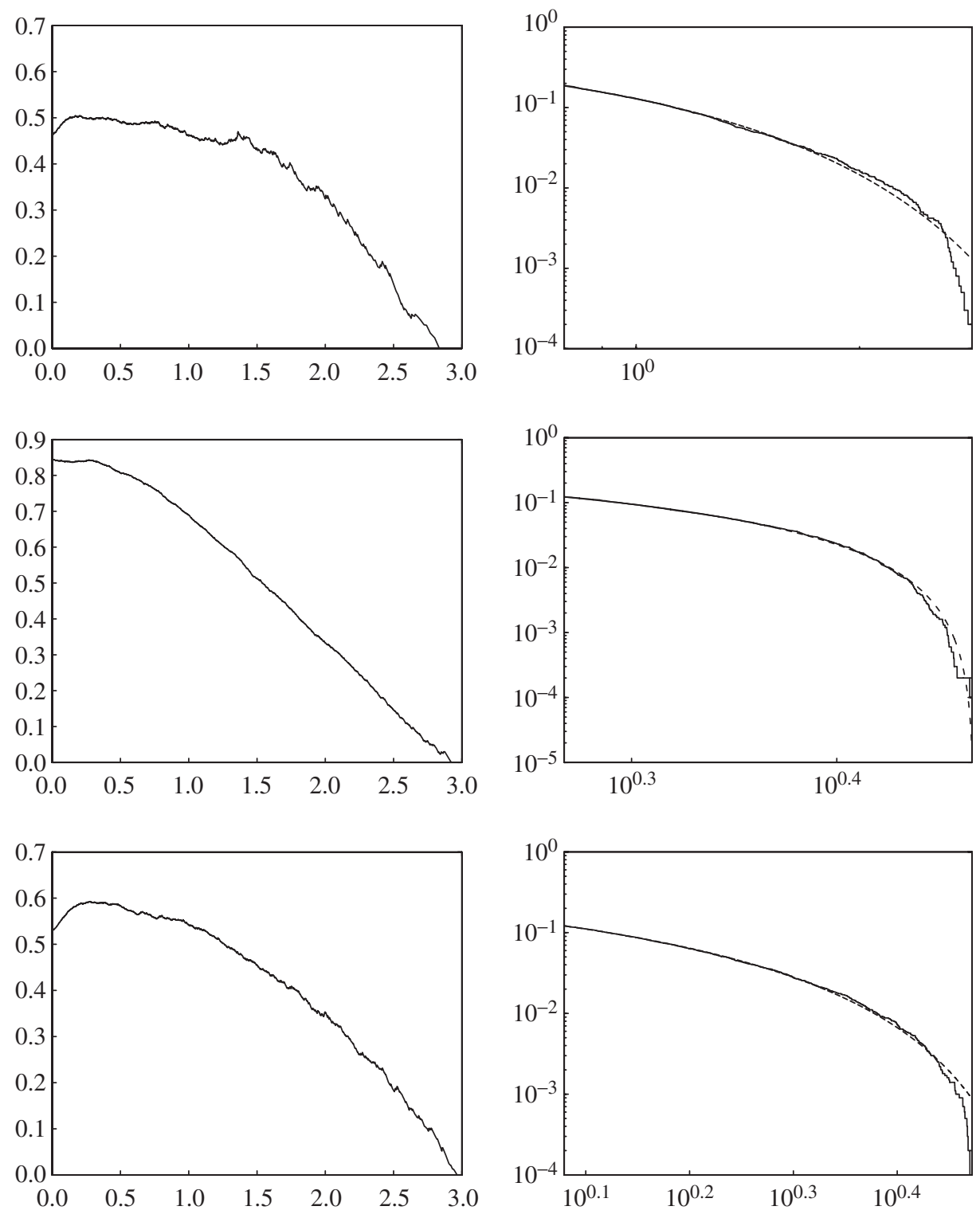

Figure 3: Length of the chord $L^{0}$ at threshold $\gamma=1$ for examples 1, 2, and 3 (in order from top to bottom) of the covariance function $\rho$. Left: the empirical mean excess plots. Right: log-log plots of the tails of the sample (solid lines) and approximating GPD (dashed lines) distribution functions. The parameters are given in the top half of Table 3.

chosen thresholds, and for the three covariance functions $\rho$. We note that a statistical test of an exponential decay could also be applied to the tails of the chord length distributions; see, e.g. [4].

In conclusion our empirical analysis appears to indicate light tails of the chord length, and the result does not seem to be sensitive to the choice of a threshold or a covariance function. To understand this phenomenon, we turn now to a probabilistic analysis. 

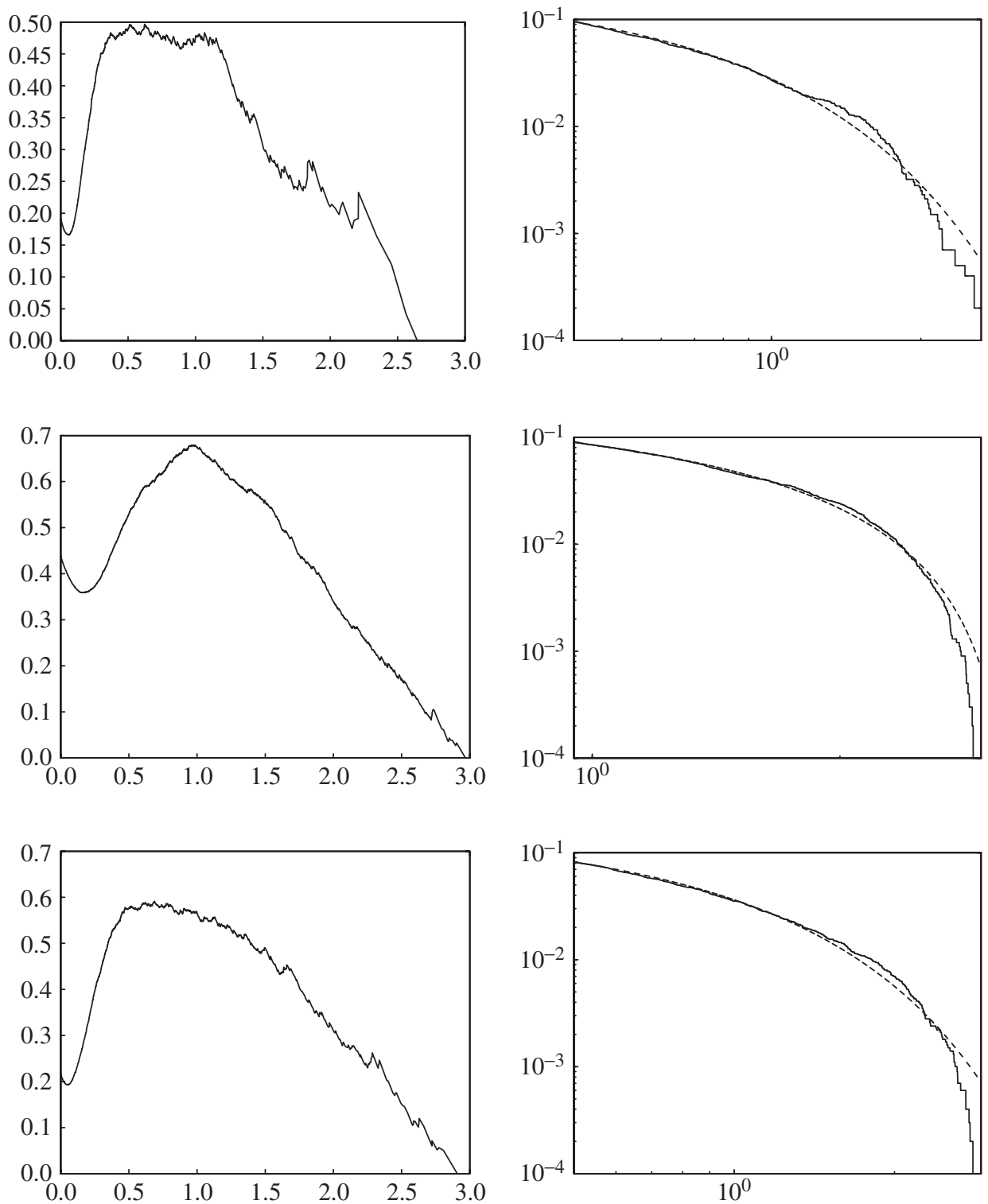

FIGURE 4: Length of the chord $L^{1}$ at threshold $\gamma=1$ for examples 1, 2, and 3 (in order from top to bottom) of the covariance function $\rho$. Left: the empirical mean excess plots. Right: log-log plots of the tails of the sample (solid lines) and approximating GPD (dashed lines) distribution functions. The parameters are given in the bottom half of Table 3.

\section{Theoretical bounds for the tail of chord length distributions}

In this section we derive upper bounds for the tail of the chord length distribution for certain families of stochastic processes, mostly stationary Gaussian processes, with appropriate assumptions on the memory of the process (on the covariance function if the process is 
Gaussian). We will see that, under mild assumptions, a faster than polynomially fast rate of decay of the chord length distribution is obtained while stronger assumptions lead to an exponentially fast rate of decay. The bounds we obtain provide an explanation of the numerical results discussed in Section 2.

We start by introducing the terminology related to the speed of decay of the tail of distributions.

Definition. Let $F$ be a mapping from $[0, \infty)$ into $[0, \infty)$. We say that $F$ decays exponentially fast if there exist $\theta>0$ and $T_{0}>0$ such that

$$
F(t) \leq \mathrm{e}^{-\theta t} \quad \text { for all } t \geq T_{0}
$$

and decays faster than polynomially fast if

$$
\lim _{t \rightarrow \infty} t^{\beta} F(t)=0 \quad \text { for all } \beta>0 .
$$

Clearly, the two notions are not mutually exclusive.

Let $X=\left(X_{t}, t \geq 0\right)$ be a continuously differentiable, strictly stationary process with mean 0 and variance 1 .

There is a natural dichotomy between the behavior of chord lengths if the starting point $X_{0}$ is below or above level $\gamma$. To account for this, we will write, for any chord length $L^{k}$ and any $t \geq 0$,

$$
\mathrm{P}\left(L^{k}>t\right)=\mathrm{P}\left(L^{k}>t, X_{0}>\gamma\right)+\mathrm{P}\left(L^{k}>t, X_{0} \leq \gamma\right) .
$$

Then we estimate each term on the right-hand side, essentially in the same way. Moreover, we invariably use a discretization of time.

\subsection{General inequalities and example}

We start with two general inequalities. The first inequality will allow us to derive asymptotic upper bounds, under the stationary probability measure $\mathrm{P}$, on the tail of the distribution of any chord length $L^{k}$ from those obtained for $L^{0}$. The second inequality will make it possible to extend the asymptotic upper bounds on the tail of the chord length distribution from the stationary probability $\mathrm{P}$ to the Palm probability measures.

Finally, we exhibit a simple example in which the underlying process $X$ has memory that does not last longer than $m$ units of time. This example already contains the key idea used in the sequel.

\subsubsection{Rate of decay of the tail of the distribution of the kth chord length.}

Proposition 3.1. For any $k \geq 0$ and any $t \geq 0$, we have

$$
\mathrm{P}\left(L^{k}>t\right) \leq 2^{k} \mathrm{P}\left(L^{0}>\frac{t}{(k+1) !}\right) .
$$

In particular, if the tail of the distribution of $L^{0}$ decays exponentially fast, or faster than polynomially fast, then the same is respectively true for the tail of the distribution of $L^{k}$ for any $k \geq 0$. 
Proof. The inequality can be easily proved by induction on $k$, beginning with $k=0$, using the decomposition

$$
\begin{aligned}
\mathrm{P}\left(L^{k}>t\right)= & \mathrm{P}\left(\text { there exist } j \in\{0, \ldots, k-1\}, L^{j}>\frac{t}{k+1}, L^{k}>t\right) \\
& +\mathrm{P}\left(\text { for all } j \in\{0, \ldots, k-1\}, L^{j} \leq \frac{t}{k+1}, L^{k}>t\right) \\
\leq & \sum_{j=0}^{k-1} \mathrm{P}\left(L^{j}>\frac{t}{k+1}\right)+\mathrm{P}\left(\bigcap_{s \in(k t /(k+1), t)}\left(X_{s}>\gamma\right) \cup \bigcap_{s \in(k t /(k+1), t)}\left(X_{s}<\gamma\right)\right),
\end{aligned}
$$

and noting that, by stationarity, the last term on the right-hand side above is less than or equal to $\mathrm{P}\left(L^{0}>t /(k+1)\right)$.

Therefore, from now on, we will derive bounds for only the tail of the distribution of the initial chord length $L=L^{0}$.

3.1.2. Chord lengths under the Palm probability measures. In practice it is of special interest to study the chord length distribution from the moment a level crossing occurs. For that purpose, we introduce the so-called Palm probability measures. For instance, to study the chord length distributions occurring after an upcrossing of the level $\gamma$, we introduce the Palm probability measure (see, for instance, [1] and [6, p. 224]) defined by

$$
\mathrm{P}_{0^{+}}(A)=\frac{1}{\mu} \lim _{\tau \rightarrow 0^{+}} \frac{1}{\tau} \mathrm{P}(A \cap(U(-\tau, 0) \geq 1)), \quad A \in \mathcal{F},
$$

where $U(s, t)$ denotes the number of upcrossings in the interval $(s, t)$ of the level $\gamma$ by the process $X$. Furthermore, $\mu=\mathrm{E}[U(0,1)]$ is assumed to be finite; recall (see [6, p. 200]) that, under our assumptions,

$$
\mathrm{P}(U(0, t) \geq 1)=\mu t+o(t) \quad \text { as } t \rightarrow 0 .
$$

The Palm probability measures $\mathrm{P}_{0^{-}}$, describing the behavior of the process after a downcrossing, and $\mathrm{P}_{0}$, describing the behavior of the process after a crossing, are defined analogously.

The distributions of $L$ under $\mathrm{P}$ and under $\mathrm{P}_{0^{+}}$are linked by

$$
\mathrm{P}_{0^{+}}(L>t)=-\frac{1}{\mu} \frac{\partial}{\partial t} \mathrm{P}\left(X_{0}<\gamma, L>t\right)
$$

see, e.g. [1], [6, p. 225], and [22, p. 47].

Even though the distributions of the chord lengths under the Palm probability measures are of main interest, they are difficult to evaluate. Fortunately, we have the following result.

Proposition 3.2. For any $t>0$, we have

$$
\mathrm{P}_{0^{+}}(L>2 t) \leq \frac{1}{t \mu} \mathrm{P}(L>t) .
$$

In particular, if the tail distribution of $L$ under the stationary probability measure $\mathrm{P}$ decays exponentially fast, or faster than polynomially fast, then the same is respectively true under the Palm probability measure $\mathrm{P}_{0^{+}}$. 
Proof. The proof follows by writing

$$
\mathrm{P}_{0^{+}}(L>2 t) \leq \frac{1}{t} \int_{t}^{2 t} \mathrm{P}_{0^{+}}(L>s) \mathrm{d} s
$$

and using (3.2).

A similar argument gives the corresponding bounds for the other Palm probabilities:

$$
\mathrm{P}_{0^{-}}(L>2 t) \leq \frac{1}{t \mu} \mathrm{P}(L>t) \quad \text { and } \quad \mathrm{P}_{0}(L>2 t) \leq \frac{1}{2 t \mu} \mathrm{P}(L>t) .
$$

3.1.3. Example: an $m$-dependent process $X$. Recall that a stochastic process $X$ is $m$-dependent if $X_{s}$ and $X_{t}$ are independent whenever $|t-s|>m$. For second-order stationary processes, the $m$-dependence implies that the covariance function of the process vanishes after lag $m$, while, for stationary Gaussian processes, the converse statement is true as well. Processes with such covariance functions are commonly used in simulations (see, e.g. [18]). It is the case for our example 1 in the simulation part (see Section 2.1).

Proposition 3.3. Assume that $X$ is an $m$-dependent stationary process such that $\mathrm{P}\left(X_{0}>\right.$ $\gamma)>0$ and $\mathrm{P}\left(X_{0}<\gamma\right)>0$. Then the tail of the distribution of the chord length $L$ decays exponentially fast.

Proof. Let $t>m^{\prime}>m$ and $n=\left[t / m^{\prime}\right] \geq 1$. Clearly,

$$
\begin{aligned}
\mathrm{P}\left(L>t, X_{0}<\gamma\right) & \leq \mathrm{P}\left(L>n m^{\prime}, X_{0}<\gamma\right) \\
& \leq \prod_{k=0}^{n} \mathrm{P}\left(X_{k m^{\prime}}<\gamma\right) \\
& =\mathrm{P}\left(X_{0}<\gamma\right)^{n+1} \\
& \leq \exp \left(t \frac{\log \mathrm{P}\left(X_{0}<\gamma\right)}{m^{\prime}}\right) .
\end{aligned}
$$

We deduce that

$$
\mathrm{P}(L>t) \leq 2 \mathrm{e}^{-\theta t} \text { for all } t>m^{\prime},
$$

with

$$
\theta=\frac{1}{m^{\prime}} \max \left\{\left|\log \mathrm{P}\left(X_{0}<\gamma\right)\right|,\left|\log \mathrm{P}\left(X_{0} \geq \gamma\right)\right|\right\} .
$$

This implies the exponentially fast rate of decay of the tail.

We mention that the above result remains valid when the $m$-dependence property of the process $X$ is replaced by certain strong mixing properties such as $\psi$-mixing (see [2] for a precise definition).

We now consider the case of stationary Gaussian processes under weaker assumptions than the $m$-dependence considered above.

\subsection{Gaussian processes with vanishing memory}

Assume that the covariance function $\rho$ of a stationary Gaussian process $X$ tends to 0 at $\infty$; we will prove that the tail of the distribution of the associated chord length $L$ decays faster than polynomially fast. When having a fast rate of decay for $\rho$, the distribution $L$ will decay even 
faster. The proof is based on the application of Slepian's lemma, introducing a new Gaussian process whose covariance is compared with $\rho$. Without loss of generality, we may and will assume throughout the section that the stationary Gaussian process has zero mean and unit variance.

Theorem 3.1. Let $X$ be a stationary Gaussian process with covariance function $\rho$ such that $\rho(t) \rightarrow 0$ as $t \rightarrow \infty$.

(i) The tail of the distribution of the chord length L decays faster than polynomially fast.

(ii) Moreover, if $\rho$ decays exponentially fast then the distribution of $L$ decays exponentially fast as well.

Proof. (i) Choose $0<a<1$, and let $T$ be so large that $\rho(s) \leq a$ for all $s \geq T$. We use the discretization

$$
\mathrm{P}\left(L>t, X_{0}>\gamma\right) \leq \mathrm{P}\left(X_{j T}>\gamma, j=0,1, \ldots,\left[\frac{t}{T}\right]\right) .
$$

By Lemma 3.1 below,

$$
\begin{aligned}
\limsup _{t \rightarrow \infty} \frac{\mathrm{P}\left(L>t, X_{0}>\gamma\right)}{\log t} & \leq \limsup _{t \rightarrow \infty} \frac{\mathrm{P}\left(X_{j T}>\gamma, j=0,1, \ldots,[t / T]\right)}{\log [t / T]} \frac{\log [t]}{\log t} \\
& \leq-\frac{1-a}{a} .
\end{aligned}
$$

Letting $a \downarrow 0$ we obtain

$$
\limsup _{t \rightarrow \infty} \frac{\mathrm{P}\left(L>t, X_{0}>\gamma\right)}{\log t}=-\infty .
$$

Applying the above to the process $-X$ we also obtain

$$
\limsup _{t \rightarrow \infty} \frac{\mathrm{P}\left(L>t, X_{0}<\gamma\right)}{\log t}=-\infty,
$$

and the two statements together give us the first claim of the theorem.

Lemma 3.1. Let $0<a<1$, and let $Y_{1}, Y_{2}, \ldots$ be a centered unit variance Gaussian process such that $\operatorname{cov}\left(Y_{i}, Y_{j}\right) \leq$ a for all $i \neq j$. Then, for any $\gamma \in \mathbb{R}$,

$$
\limsup _{n \rightarrow \infty} \frac{\log \mathrm{P}\left(Y_{1}>\gamma, \ldots, Y_{n}>\gamma\right)}{\log n} \leq-\frac{1-a}{a} .
$$

Proof. Let $W_{0}, W_{1}, W_{2}, \ldots$ be independent and identically distributed standard normal random variables, and let $Z_{j}=a^{1 / 2} W_{0}+(1-a)^{1 / 2} W_{j}, j=1,2, \ldots$ Then $Z_{1}, Z_{2}, \ldots$ is a discrete-time centered unit variance Gaussian process such that $\operatorname{cov}\left(Z_{i}, Z_{j}\right)=a$ for all $i \neq j$. By the Slepian inequality (see [20]) we know that, for any $n \geq 1$ and $\gamma \in \mathbb{R}$, $\mathrm{P}\left(Y_{1}>\gamma, \ldots, Y_{n}>\gamma\right) \leq \mathrm{P}\left(Z_{1}>\gamma, \ldots, Z_{n}>\gamma\right)$. Therefore, it is enough to prove (3.3) with $\left(Y_{j}\right)$ replaced by $\left(Z_{j}\right)$.

Choose any

$$
0<\theta<\frac{1-a}{a}
$$


and write

$$
\begin{aligned}
\mathrm{P}\left(Z_{1}>\gamma, \ldots, Z_{n}>\gamma\right) & =\int_{-\infty}^{\infty} \phi(x)\left[\Psi\left(\frac{\gamma-a^{1 / 2} x}{(1-a)^{1 / 2}}\right)\right]^{n} \mathrm{~d} x \\
& =\left(\int_{x>(2 \theta \log n)^{1 / 2}}+\int_{x \leq(2 \theta \log n)^{1 / 2}}\right) \phi(x)\left[\Psi\left(\frac{\gamma-a^{1 / 2} x}{(1-a)^{1 / 2}}\right)\right]^{n} \mathrm{~d} x \\
& =: I_{1}(n)+I_{2}(n),
\end{aligned}
$$

where $\phi$ and $\Psi$ are the density and the tail of a standard normal random variable, respectively. We use the bounds

$$
\Psi(x) \leq \frac{1}{2} \mathrm{e}^{-x^{2} / 2} \quad \text { for } x \geq 0
$$

and

$$
\Psi(x)>\mathrm{e}^{-(1+\varepsilon) x^{2} / 2} \quad \text { for large enough } x \text { and any } \varepsilon>0 .
$$

First of all,

$$
I_{1}(n) \leq \Psi\left((2 \theta \log n)^{1 / 2}\right) \leq \frac{1}{2} \mathrm{e}^{-\theta \log n}=\frac{1}{2} n^{-\theta} .
$$

On the other hand, selecting $0<\varepsilon<1$ so small that

$$
\frac{\theta a(1+\varepsilon)}{1-a}<1
$$

we have, for large enough $n$,

$$
\begin{aligned}
I_{2}(n) & \leq\left[\Psi\left(\frac{\gamma-a^{1 / 2}(2 \theta \log n)^{1 / 2}}{(1-a)^{1 / 2}}\right)\right]^{n} \\
& =\left[1-\Psi\left(\frac{a^{1 / 2}(2 \theta \log n)^{1 / 2}-\gamma}{(1-a)^{1 / 2}}\right)\right]^{n} \\
& \leq\left[1-\exp \left(-\frac{(1+\varepsilon) a \theta \log n}{1-a}\right)\right]^{n} \\
& =\left(1-n^{-(1+\varepsilon) a \theta /(1-a)}\right)^{n}
\end{aligned}
$$

and, using (3.5), we see that, for large enough $n$,

$$
I_{2}(n) \leq \exp \left(-\frac{1}{2} n^{1-(1+\varepsilon) a \theta /(1-a)}\right)=o\left(n^{-\theta}\right) .
$$

Combining this bound with (3.4), we conclude that

$$
\limsup _{n \rightarrow \infty} \frac{\log \mathrm{P}\left(Z_{1}>\gamma, \ldots, Z_{n}>\gamma\right)}{\log n} \leq-\theta .
$$

Since $\theta$ can be taken arbitrarily close to $(1-a) / a$, we obtain $(3.3)$ for $\left(Z_{j}\right)$ replacing $\left(Y_{j}\right)$, as required, completing the proof of Lemma 3.1.

(ii) We continue with the proof of the second claim of Theorem 3.1. Let $T>0$ be the positive number from (3.1), the definition of the exponentially fast decay of $\rho$, and let $\theta>0$ be the corresponding exponent. We discretize the time parameter of the process $X$, defining, for 
$n \geq 0, Y_{n}=X(n T)$. Then $Y=\left(Y_{n}\right)_{n \geq 0}$ is a centered unit variance discrete-time stationary Gaussian process, whose covariance function $\rho_{Y}$ satisfies

$$
\rho_{Y}(k)=\rho(k T) \leq \mathrm{e}^{-\theta k T}, \quad k=0,1,2, \ldots
$$

For $t \geq T$, let $n=[t / T] \geq 1$, and note that

$$
\mathrm{P}(L>t) \leq \mathrm{P}\left(Y_{j}>\gamma, j=0, \ldots, n\right)+\mathrm{P}\left(Y_{j}<\gamma, j=0, \ldots, n\right) .
$$

We will only spell out the procedure for obtaining an upper bound for the first term on the right-hand side above. We will prove that there exist $\alpha_{0}>0$ and $N_{0} \geq 0$ such that

$$
\mathrm{P}\left(Y_{j}>\gamma, j=0, \ldots, n\right) \leq \mathrm{e}^{-\alpha_{0} n} \quad \text { for all } n \geq N_{0} .
$$

This will, clearly, imply that the probability $\mathrm{P}(L>t)$ decays exponentially fast.

As previously, we will use the Slepian lemma and introduce another centered unit variance discrete-time stationary Gaussian process $Z=\left(Z_{n}\right)_{n \geq 0}$, with covariance function $\rho_{Z}$ equal to the upper bound on the covariance function $\rho_{Y}$ in (3.6), i.e.

$$
\rho_{Z}(k)=r_{0}^{k}, \quad k=0,1,2, \ldots, \quad \text { with } \quad r_{0}=\mathrm{e}^{-\theta T} .
$$

Such a process $Z$ does exist; in fact, it can be represented as a causal AR(1) process defined by

$$
Z_{n+1}=r_{0} Z_{n}+\xi_{n+1}, \quad n \geq 0,
$$

where $\left(\xi_{n}\right)_{n \geq 0}$ is a Gaussian white noise $\mathcal{N}\left(\mathscr{Q}, \mathbb{E}-r_{\mathscr{Q}}^{G}\right)$. In particular, $Z$ is a Markov process. This property is important since it allows us to proceed in a similar way as in the $m$-dependent case. From the Slepian normal comparison lemma, we know that it is enough to prove (3.7) for $Z$ instead of $Y$.

The threshold $\gamma$ in (3.7) can be of any sign. Obviously, once we prove the statement for $\gamma<0$, its validity for any other $\gamma$ will follow. Nonetheless, since there is a particularly simple argument in the case $\gamma>0$ that helps to understand the trick when tackling the case $\gamma \leq 0$, we present it first.

If $\gamma>0$, we can use the simple bound

$$
\mathrm{P}\left(Z_{j}>\gamma, j=0, \ldots, n\right) \leq \mathrm{P}\left(\sum_{j=0}^{n} Z_{j}>(n+1) \gamma\right) .
$$

The random variable $\sum_{j=0}^{n} Z_{j}$ has the normal distribution $\mathcal{N}\left(\mathscr{Q}, \sigma_{n}^{G}\right)$ with $\sigma_{n}^{2} \leq n\left(1+r_{0}\right) /(1-$ $\left.r_{0}\right)$, so we obtain

$$
\mathrm{P}\left(Z_{j}>\gamma, j=0, \ldots, n\right) \leq \Psi\left(\left(n \gamma \frac{1-r_{0}}{1+r_{0}}\right)^{1 / 2}\right) \leq \mathrm{e}^{-\alpha_{0} n} \quad \text { for large enough } n,
$$

using once again the standard upper bound for $\Psi$. Note that any $\alpha_{0}<\gamma\left(1-r_{0}\right) / 2\left(1+r_{0}\right)$ can be used above. When $\gamma \leq 0$, estimate (3.8) is no longer sufficient for our purposes.

Let $\gamma^{\prime}=3-2 \gamma>0$, and consider the event

$$
A_{n}=\left\{\text { at least }\left\lceil\frac{1}{3} n\right\rceil \text { out of } Z_{0}, Z_{2}, \ldots, Z_{2 n-2} \text { are larger than } \gamma^{\prime}\right\},
$$

where $\lceil\cdot\rceil$ denotes the ceiling function. 
First, note that

$$
\begin{aligned}
\mathrm{P}\left(\left(Z_{j}>\gamma, j=0, \ldots, 2 n-1\right) \cap A_{n}\right) & \leq \mathrm{P}\left(\sum_{j=0}^{n-1} Z_{2 j}>\left(n-\left\lceil\frac{n}{3}\right\rceil\right) \gamma+\left\lceil\frac{n}{3}\right\rceil \gamma^{\prime}\right) \\
& \leq \mathrm{P}\left(\sum_{j=0}^{n-1} Z_{2 j}>n\right) .
\end{aligned}
$$

This puts us in a situation analogous to the case $\gamma>0$ above, and using the same argument, we find that, for any $\alpha_{1}<\left(1-r_{0}^{2}\right) / 2\left(1+r_{0}^{2}\right)$, there exists an $N_{1}$ such that, for all $n \geq N_{1}$,

$$
\mathrm{P}\left(\left(Z_{j}>\gamma, j=0, \ldots, 2 n-1\right) \cap A_{n}\right) \leq \mathrm{e}^{-\alpha_{1} n} .
$$

In order to estimate $\mathrm{P}\left(\left(Z_{j}>\gamma, j=0, \ldots, 2 n-1\right) \cap \overline{A_{n}}\right)$, let us introduce the sets $B_{n}, n \geq 1$, defined by

$$
\begin{array}{r}
B_{n}=\left\{\left(b_{1}, \ldots, b_{n}\right) \in \mathbb{R}^{n}: b_{j}>\gamma, j=1, \ldots, n, \text { and at most }\left\lceil\frac{1}{3} n\right\rceil-1\right. \\
\text { are larger the } \left.b_{j} \mathrm{~s} \gamma^{\prime}\right\} .
\end{array}
$$

For every $n \geq 1$, we define a function on $\mathbb{R}^{n}$ by

$$
Q_{n}\left(z_{0}, \ldots, z_{2 n-2}\right)=\mathrm{P}\left(Z_{2 j+1}>\gamma, j=0, \ldots, n-1 \mid Z_{2 j}=z_{2 j}, j=0, \ldots, n-1\right)
$$

for $\left(z_{0}, \ldots, z_{2 k}, \ldots, z_{2 n-2}\right) \in \mathbb{R}^{n}$, in the sense of the usual continuous conditional probabilities, and write

$$
\begin{aligned}
& \mathrm{P}\left(\left\{Z_{j}\right.\right.\left.>\gamma, j=0, \ldots, 2 n-1\} \cap \overline{A_{n}}\right) \\
& \quad=\int_{B_{n}} Q_{n}\left(z_{0}, \ldots, z_{2 n-2}\right) \phi_{n}\left(z_{0}, \ldots, z_{2 n-2}\right) \mathrm{d} z_{0} \cdots \mathrm{d} z_{2 n-2},
\end{aligned}
$$

where $\phi_{n}$ is the joint probability density function of $\left(Z_{0}, Z_{2}, \ldots, Z_{2 n-2}\right)$.

Given $\left(z_{0}, z_{1}, \ldots, z_{2 n-2}, z_{2 n-1}\right)$ such that the vector of the even-numbered coordinates $\left(z_{0}, z_{2}, \ldots, z_{2 n-2}\right)$ is in $B_{n}$, the latter vector has at least $n-\lceil n / 3\rceil+1$ coordinates which are strictly smaller than $\gamma^{\prime}$. Elementary counting shows that there are at least $\lceil n / 3\rceil$ odd numbers $j_{1}<j_{2}<\cdots<j_{\lceil n / 3\rceil}$ in the set $\{1,3, \ldots, 2 n-3\}$ such that, for every $k \in\{1, \ldots,\lceil n / 3\rceil\}$,

$$
z_{j_{k}-1}<\gamma^{\prime} \text { and } z_{j_{k}+1}<\gamma^{\prime}
$$

It follows from the Markov property of the process $Z$ that

$$
\begin{aligned}
Q_{n}\left(z_{0}, \ldots, z_{2 n-2}\right) & \leq \mathrm{P}\left(Z_{j_{1}}>\gamma, Z_{j_{2}}>\gamma, \ldots, Z_{j_{\lceil n / 3\rceil}}>\gamma \mid Z_{2 j}=z_{2 j}, j=0, \ldots, n-1\right) \\
& =\prod_{k=1}^{\lceil n / 3\rceil} \mathrm{P}\left(Z_{j_{k}}>\gamma \mid Z_{j_{k}-1}=z_{j_{k}-1}, Z_{j_{k}+1}=z_{j_{k}+1}\right) .
\end{aligned}
$$

Recall that all $z$-values in the conditions are smaller than $\gamma^{\prime}$.

Given $\left(Z_{j_{k}-1}=z_{j_{k}-1}, Z_{j_{k}+1}=z_{j_{k}+1}\right)$, the random variable $Z_{j_{k}}$ has the normal distribution $\mathcal{N}\left(\mu_{k}, \sigma^{G}\right)$ with

$$
\mu_{k}=\frac{r_{0}}{1+r_{0}^{2}}\left(z_{j_{k}-1}+z_{j_{k}+1}\right)<\frac{2 \gamma^{\prime} r_{0}}{1+r_{0}^{2}} \quad \text { and } \quad \sigma^{2}=\frac{1+r_{0}^{4}}{\left(1+r_{0}^{2}\right)^{2}}>0 .
$$


Therefore,

$$
Q_{n}\left(z_{0}, \ldots, z_{2 n-2}\right) \leq\left(\Psi\left(\frac{\gamma\left(1+r_{0}^{2}\right)-2 \gamma^{\prime} r_{0}}{\sqrt{1+r_{0}^{4}}}\right)\right)^{\lceil n / 3\rceil},
$$

and it follows from (3.10) that, for all $n \geq 1$,

$$
\mathrm{P}\left(\left\{Z_{0}>\gamma, \ldots, Z_{2 n-1}>\gamma\right\} \cap \overline{A_{n}}\right) \leq \mathrm{e}^{-\alpha_{2} n},
$$

with $\alpha_{2}=\Psi\left(\left(\gamma\left(1+r_{0}^{2}\right)-2 \gamma^{\prime} r_{0}\right) / \sqrt{1+r_{0}^{4}}\right)$. Combining this bound with (3.9), shows that there exists $N_{2}$ such that, for all $n \geq N_{2}$,

$$
\mathrm{P}\left(Z_{0}>\gamma, \ldots, Z_{2 n-1}>\gamma\right) \leq \mathrm{e}^{-\alpha_{3} n},
$$

where we can use any $\alpha_{3}<\min \left(\alpha_{1}, \alpha_{2}\right)$. This proves (3.7) with any $\alpha_{0}<\alpha_{3} / 2$ and some large enough $N_{0}$. This completes the proof of Theorem 3.1.

Since a stationary Gaussian process is mixing if and only if its covariance function converges to 0 (see [5]), Theorem 3.1(i) states that any mixing stationary Gaussian process has the property that the distribution of $L$ decays faster than polynomially fast. We do not know at the moment if this property holds also for non-Gaussian mixing stationary processes. Nevertheless, the conclusion holds under a stronger dependence condition when assuming that a non-Gaussian process is $r$-mixing (see [8]).

\section{3. $r$-mixing processes}

Recall that the $r$-mixing coefficients of a process $X$ are defined by

$$
r_{T}=r\left(A, B_{T}\right)=\sup _{W_{1} \in L_{X}^{2}(A), W_{2} \in L_{X}^{2}\left(B_{T}\right)}\left|\operatorname{corr}\left(W_{1}, W_{2}\right)\right|, \quad T>0,
$$

where $A=(-\infty, 0]$ and $B_{T}=[T, \infty)$, and, for $D \subset \mathbb{R}, L_{X}^{2}(D)$ denotes the closure in $L^{2}(\Omega)$ of $\operatorname{span}\left\{X_{t}, t \in D\right\}$.

In particular, for any $T>0$ and events $C_{1}$ and $C_{2}$ such that $W_{1}=\mathbf{1}_{C_{1}} \in L_{X}^{2}(A)$ and $W_{2}=\mathbf{1}_{C_{2}} \in L_{X}^{2}\left(B_{T}\right)$, we have

$$
r_{T} \geq \frac{\left|\mathrm{P}\left(C_{1} \cap C_{2}\right)-\mathrm{P}\left(C_{1}\right) \mathrm{P}\left(C_{2}\right)\right|}{\left(\mathrm{P}\left(C_{1}\right) \mathrm{P}\left(\overline{C_{1}}\right) \mathrm{P}\left(C_{2}\right) \mathrm{P}\left(\overline{C_{2}}\right)\right)^{1 / 2}} .
$$

We will say that $X$ is an $r$-mixing process if it satisfies

$$
\lim _{T \rightarrow+\infty} r_{T}=0
$$

Theorem 3.2. Assume that $X$ is a stationary $r$-mixing process such that $\mathrm{P}\left(X_{0}>\gamma\right)>0$ and $\mathrm{P}\left(X_{0}<\gamma\right)>0$. Then the tail of the distribution of the chord length $L$ decays faster than polynomially fast.

Proof. Fix $T>0$ large enough so that $r_{T}<\min \left(\mathrm{P}\left(X_{0}<\gamma\right), \mathrm{P}\left(X_{0}>\gamma\right)\right)$. Define, for $n \geq 0, Y_{n}=X(n T)$, and consider the discrete-time process $Y=\left(Y_{n}\right)_{n \geq 0}$.

For $n \geq 0$, we apply (3.11) with $C_{1}=\left\{X\left(-\left(2^{n}-1\right) T\right)>\gamma, \ldots, X(0)>\gamma\right\}$ and $C_{2}=\left\{X(T)>\gamma, \ldots, X\left(2^{n} T\right)>\gamma\right\}$. By stationarity,

$$
\begin{aligned}
\mathrm{P}\left(Y_{0}\right. & \left.>\gamma, \ldots, Y_{2^{n+1}-1}>\gamma\right) \\
& =\mathrm{P}\left(\left\{Y_{0}>\gamma, \ldots, Y_{2^{n}-1}>\gamma\right\} \cap\left\{Y_{2^{n}}>\gamma, \ldots, Y_{2^{n+1}-1}>\gamma\right\}\right) \\
& \leq\left(\mathrm{P}\left(Y_{0}>\gamma, \ldots, Y_{2^{n}-1}>\gamma\right)\right)^{2}+r_{T} \mathrm{P}\left(Y_{0}>\gamma, \ldots, Y_{2^{n}-1}>\gamma\right) .
\end{aligned}
$$


Defining $p_{n}=\mathrm{P}\left(Y_{0}>\gamma, \ldots, Y_{2^{n}-1}>\gamma\right)$ for $n \geq 0$, we see that

$$
p_{n+1} \leq p_{n}^{2}+r_{T} p_{n}, \quad n=0,1, \ldots,
$$

which implies that $p_{n} \rightarrow 0$ as $n \rightarrow \infty$ (if $l=\lim _{n \rightarrow \infty} p_{n} \neq 0$ then $l>p_{0}$, which would contradict ( $\left.p_{n}\right)$ decreasing). Therefore, there exists $N_{0} \geq 0$ such that, for all $n \geq N_{0}$, we have $p_{n} \leq r_{T}$. Thus,

$$
p_{n+1} \leq 2 r_{T} p_{n} \text { for all } n \geq N_{0},
$$

and, hence,

$$
p_{n} \leq c_{0}\left(2 r_{T}\right)^{n} \quad \text { with } \quad c_{0}=p_{N_{0}}\left(2 r_{T}\right)^{-N_{0}}>0 \quad \text { for all } n \geq N_{0} .
$$

Now let $t>2^{N_{0}} T$ be so large that $n=\left[\log _{2}(t / T)\right] \geq N_{0}$ and remark that $r_{T}<\frac{1}{2}$. We have, with $\log _{2}$ denoting the logarithm in base 2 ,

$$
\mathrm{P}\left(L>t, X_{0}>\gamma\right) \leq \mathrm{P}\left(Y_{0}>\gamma, \ldots, Y_{2^{n}-1}>\gamma\right) \leq c_{0}\left(2 r_{T}\right)^{n} \leq c_{0}^{\prime} t^{-\left|\log _{2}\left(2 r_{T}\right)\right|}
$$

for a certain constant $c_{0}^{\prime}>0$ depending only on $N_{0}$ and $T$. We deduce that

$$
\varlimsup_{t \rightarrow \infty} \frac{\log \mathrm{P}\left(L>t, X_{0}>\gamma\right)}{\log t} \leq-\left|\log _{2}\left(2 r_{T}\right)\right| .
$$

Since $r_{T} \rightarrow 0$ as $T \rightarrow \infty$, we conclude that

$$
\lim _{t \rightarrow \infty} \frac{\log \mathrm{P}\left(L>t, X_{0}>\gamma\right)}{\log t}=-\infty .
$$

Hence, $t^{\beta} \mathrm{P}\left(L>t, X_{0}>\gamma\right) \rightarrow 0$ as $t \rightarrow \infty$, for any $\beta>0$.

An analogous result holds for $\mathrm{P}\left(L>t, X_{0} \leq \gamma\right)$. Therefore, the distribution of $L$ decays faster than polynomially fast.

Recall that a stationary Gaussian process with a covariance function which decays in a polynomial way is $r$-mixing (see [5]). So an immediate corollary of Theorem 3.2 is the fact that, for Gaussian processes with polynomially decaying covariance function, the distribution of the chord length $L$ decays faster than polynomially fast, a result obtained in Theorem 3.1(i) under a weaker assumption.

\section{Conclusion}

We have established theoretical results on the rate of decay of the tail of the distribution of the chord lengths, depending on the memory in the thresholded process. In the case of stationary Gaussian processes, the memory is expressed via the covariance function. The results agree with the empirical results obtained by statistical analysis of simulated processes. It shows that, as soon as one deals with a thresholded stationary process whose covariance decays to 0 , a rapidly decreasing decay of the chord length distribution has to be expected. Consequently, it seems hopeless to use such a thresholded process as a model for real data when, for instance, a power-like decay of the chord length distribution is observed.

We focused on the asymptotic behavior of the tails of the distributions and did not try to derive the best constants in the upper bounds for such tails. It may be possible to refine these bounds and relate them to the threshold. It may further be possible to explore how the bounds 
change from one chord length to the next. This will be the subject of a future work. We also plan to investigate what memory properties of the thresholded process imply lower bounds on the tails of the chord length distributions.

We hope that our work will also contribute to the study of the $D$-dimensional Boolean models for $D>1$. For instance, if $L^{*}$ is the spherical contact distance of the phase containing the origin, upper bounds for $\mathrm{P}\left(L^{*}>t\right)$ can be obtained from our results since

$$
L^{*}=\sup \{R>0: B(0, R) \subset 0 \text {-phase }\}=\inf _{\alpha \in[0,2 \pi)} L(\alpha),
$$

where the 0-phase is the phase containing the origin and $L(\alpha)$ denotes the initial chord length in the direction $\alpha$.

\section{Acknowledgements}

This work was partially supported by the French grant 'mipomodim' ANR-05-BLAN-0017. Samorodnitsky's research was also partially supported by the ARO grant W911NF-07-1-0078 at Cornell University.

\section{References}

[1] Baccelli, F. and Brémaud, P. (2003). Elements of Queueing Theory. Springer, Berlin.

[2] Bradley, R. C. (1986). Basic properties of strong mixing properties. In Dependence in Probability and Statistics (Oberwolfach, 1985; Progr. Prob. Statist. 11), Birkhäuser, Boston, MA, pp. 165-192.

[3] Bron, F. And Jeulin, D. (2004). Modelling a food microstructure by random sets. Image Anal. Stereology 23, $33-44$.

[4] Chaouche, A. and Bacro, J.-N. (2004). A statistical test procedure for the shape parameter of a generalized Pareto distribution. Comput. Statist. Data Anal. 45, 787-803.

[5] Cornfield, I. P., Fomin, S. V. And Sinai, Y. G. (1982). Ergodic Theory. Springer, New York.

[6] Cramér, H. and Leadbetter, M. R. (1967). Stationary and Related Stochastic Processes. Sample Function Properties and Their Applications. John Wiley, New York.

[7] Dietrich, C. R. And Newsam, G. N. (1997). Fast and exact simulation of stationary Gaussian processes through circulant embedding of the covariance matrix. SIAM J. Sci. Comput. 18, 1088-1107.

[8] Doukhan, P. (1994). Mixing (Lecture Notes Statist. 85). Springer, New York.

[9] Embrechts, P., Klüppelberg, C. and Mikosch, T. (2003). Modelling Extremal Events. Springer, Berlin.

[10] Estrade, A., Iribarren, I. And Kratz, M. (2008). Chord-distribution functions and Rice formulae. Application to random media. Available at http://hal.archives-ouvertes.fr/hal-00161806/fr/.

[11] Gneiting, T. et al. (2006). Fast and exact simulation of large Gaussian lattice systems in $\mathbb{R}^{2}$ : exploring the limits. J. Comput. Graph. Statist. 15, 483-501.

[12] Hosking, J. R. M. and Wallis, J. R. (1987). Parameter and quantile estimation for the generalized Pareto distribution. Technometrics 29, 339-349.

[13] Nott, D. J. And Wilson, R. J. (2000). Multi-phase image modelling with excursion sets. Signal Processing 80, 125-139.

[14] Pickands, J. (1975). Statistical inference using extreme order statistics. Ann. Statist. 3, 119-131.

[15] Roberts, A. P. AND Teubner, M. (1995). Transport properties of heterogeneous materials derived from Gaussian random fields: bounds and simulation. Phys. Rev. E 51, 4141-4154.

[16] Roberts, A. P. and Torquato, S. (1999). Chord-distribution functions of three-dimensional random media: approximate first-passage times of Gaussian processes. Phys. Rev. E 59, 4953-4963.

[17] Schlather, M. (2001). Simulation and analysis of random fields. R News 1, 18-20.

[18] Schlather, M. (2009). Reference manual for the Random Fields packages in R. Available at http://cran.rproject.org/web/packages/RandomFields/.

[19] Schüth, F., Sing, K. S. W. And Weitkamp, J. (eds) (2002). Handbook of Porous Solids. John Wiley, New York.

[20] Slepian, D. (1962). The one-sided barrier problem for Gaussian noise. Bell System Tech. J. 41, 463-501,

[21] Stoyan, D., Kendall, W. S. and Mecke, J. (1995). Stochastic Geometry and Its Applications. John Wiley, Chichester.

[22] Torquato, S. (2002). Random Heterogeneous Materials. Springer, New York. 